ARTICLE

Received 26 Sep 2014 | Accepted 3 Mar 2014 | Published 14 Apr $2014 \quad$ DOl: 10.1038/ncomms4532

\title{
Joint estimation of phase and phase diffusion for quantum metrology
}

Mihai D. Vidrighin ${ }^{1,2}$, Gaia Donati ${ }^{2}$, Marco G. Genoni ${ }^{1,3}$, Xian-Min Jin²,4, W. Steven Kolthammer ${ }^{2}$, M.S. Kim¹, Animesh Datta ${ }^{2}$, Marco Barbieri² \& lan A. Walmsley ${ }^{2}$

Phase estimation, at the heart of many quantum metrology and communication schemes, can be strongly affected by noise, whose amplitude may not be known, or might be subject to drift. Here we investigate the joint estimation of a phase shift and the amplitude of phase diffusion at the quantum limit. For several relevant instances, this multiparameter estimation problem can be effectively reshaped as a two-dimensional Hilbert space model, encompassing the description of an interferometer probed with relevant quantum statessplit single-photons, coherent states or NOON states. For these cases, we obtain a trade-off bound on the statistical variances for the joint estimation of phase and phase diffusion, as well as optimum measurement schemes. We use this bound to quantify the effectiveness of an actual experimental set-up for joint parameter estimation for polarimetry. We conclude by discussing the form of the trade-off relations for more general states and measurements.

\footnotetext{
${ }^{1}$ QOLS, Blackett Laboratory, Imperial College London, London SW7 2BW, UK. ${ }^{2}$ Clarendon Laboratory, Department of Physics, University of Oxford, Oxford OX1 3PU, UK. ${ }^{3}$ Department of Physics \& Astronomy, University College London, Gower Street, London WC1E 6BT, UK. ${ }^{4}$ Department of Physics and Astronomy, Shanghai Jiao Tong University, Shanghai 200240, China. Correspondence and requests for materials should be addressed to M.D.V. (email: m.vidrighin11@imperial.ac.uk).
} 
Ex: fficient sensing and imaging of samples that cannot be exposed to high field intensities require the optimization of the amount of information acquired from each run of an experiment. Judiciously designed quantum strategies can lead to significant improvements of sensitivities, when compared with classical strategies with probes of equivalent energy content ${ }^{1}$. Phase estimation illustrates well the advantages of quantum metrology, with wide-ranging practical applications ${ }^{2-7}$. Variations of many physical properties such as weak fields ${ }^{8}$ displacements or changes in concentration ${ }^{9,10}$ can be efficiently observed as phase shifts.

A central aspect of sensing in a real scenario is the interaction between the system and the environment. When one takes into account this coupling, the promised quantum enhancement is likely to be lost ${ }^{11}$. This has been extensively shown in the case of a lossy interferometer ${ }^{12-15}$ while, more recently, theoretical and experimental efforts have been directed at studying the limits of phase estimation in the presence of phase diffusion ${ }^{16-20}$. Phasediffusive noise describes fluctuations in the modes of an interferometer, which can be modelled as random phase-kicks. This process reduces the visibility of interference, directly affecting precision measurements as, for instance, in Ramsey interferometry. The works cited above studied the limits of phase estimation given a known amount of phase diffusion.

However, in several physical processes, such as path length fluctuations of a stabilized interferometer, thermal fluctuations of an optical fibre and weak coupling of the probed system to the environment, phase and phase diffusion may vary in time. Consequently, phase estimation relying on past estimates of the magnitude of phase diffusion may lead to inaccuracies. A more accurate and robust solution consists in estimating the phase shift and the phase diffusion in a simultaneous, joint scheme. This allows for the monitoring of both parameters on the relevant timescale avoiding systematic effects. Moreover, the consideration of joint estimation for phase and noise amplitude leads to a fair accounting of resources, eliminating the need for precise calibration before the estimation. These represent important motivations for exploring the fundamental limits of this multiparameter scenario. Similar investigations have received considerable attention recently ${ }^{21-32}$.

In this paper, we study theoretically as well as experimentally the simultaneous estimation of phase shift and diffusion using quantum states that can be effectively described in a twodimensional Hilbert space (as qubits). This provides a description of two-arm interferometry with some of the relevant probe states in quantum metrology-coherent and N00N states ${ }^{33}$. We find that the quantum precision limit for the estimation of each of the parameters cannot be reached for both parameters simultaneously. We derive a trade-off relation that must be obeyed by the statistical variances attainable through any physical measurement and use it to identify a double-homodyne set-up as an optimal choice for phase and phase diffusion measurements for the considered set of probe states. We show this trade-off via a tunable measurement, which we characterize by quantum detector tomography. Finally, we analyse the applicability of our bounds for more general states and measurements. To this end, we present the results of numerical searches for optimum measurements acting on Holland-Burnett states ${ }^{2}$, as well as on a pair of qubits. We also present the performance of the double-homodyne measurement set-up that we identified as optimal, in the presence of losses.

\section{Results}

Quantum estimation theory. Fisher information (FI) provides an asymptotic measure of the amount of information on the parameters of a system that is acquired by performing a measurement on it. For parameters $\lambda$, in terms of the probabilities associated to the measurement results $p_{n}=p_{n}(\lambda)$, the FI matrix elements $\operatorname{read}^{34} F_{i, j}=\sum_{n} p_{n} \frac{\partial^{2}}{\partial \lambda_{i} \partial \lambda_{j}} \log \left(p_{n}\right)=\sum_{n} \frac{1}{p_{n}} \frac{\partial p_{n}}{\partial \lambda_{i}} \frac{\partial p_{n}}{\partial \lambda_{j}}$. The Cramér-Rao bound states that, for all unbiased estimators $\hat{\lambda}$, the expected covariance matrix with elements defined as $\gamma_{i, j}=\left\langle\hat{\lambda}_{i} \hat{\lambda}_{j}\right\rangle-\left\langle\hat{\lambda}_{i}\right\rangle\left\langle\hat{\lambda}_{j}\right\rangle$ satisfies

$$
\gamma \geq(M \mathbf{F})^{-1}
$$

where $M$ is the number of experimental runs. The Cramér-Rao bound is saturated asymptotically by the maximum likelihood estimator, upon elimination of systematic errors. FI provides a useful indicator of the optimality of a given experiment and constitutes a useful tool for designing measurements with the goal of minimizing statistical errors.

For a set of probabilities that originated from measurements on a quantum system, the ultimate limit on the covariance matrix is set by the quantum Cramér-Rao bound in terms of the quantum FI (QFI) matrix ${ }^{35,36}$. Introducing the symmetric logarithmic derivative (SLD) operator $L_{j}$ for parameter $\lambda_{j}$, obeying $2 \partial_{\lambda_{j}} \rho=L_{j} \rho+\rho L_{j}$, the QFI matrix is defined as $H_{i j}=\operatorname{Re}\left[\operatorname{Tr}\left[\rho L_{i} L_{j}\right]\right]$ and it bounds the FI matrix corresponding to any particular measurement: $\mathbf{H} \geq \mathbf{F}$. For a single parameter, the ultimate bound can always be achieved choosing the measurement given by the eigenvectors of the SLD operator. In the case of a multi-parameter problem, if the SLDs corresponding to different parameters do not commute then the FI values for the two parameters are maximized by incompatible measurements.

Interferometry with phase diffusion. We consider an interferometer with phase difference $\phi$ between its two arms. The annihilation operators corresponding to each arm are labeled $\hat{a}$ and $\hat{b}$. Different physical processes lead to phase diffusion and the corresponding channel can be modelled as a random phase shift distributed according to a normal distribution of width $\Delta$, called the noise amplitude. Acting on a mode $a$ with initial state $\rho_{\text {in }}$, the phase diffusion channel yields

$$
\rho=\mathcal{N}_{\Delta}\left(\rho_{\text {in }}\right)=\frac{1}{\sqrt{2 \pi} \Delta} \int \mathrm{d} \xi e^{-\frac{\xi^{2}}{2 \Delta^{2}}} U_{\xi} \rho_{\text {in }} U_{\xi}^{\dagger}
$$

where $U_{\xi}=\exp \left(i \xi \hat{a}^{\dagger} \hat{a}\right)$ is the phase shift operator. In the Fock basis, the result is the exponential erasing of the off-diagonal elements of the density matrix:

$$
\mathcal{N}_{\Delta}(|n\rangle\langle m|)=e^{-\Delta^{2}(n-m)^{2}}|n\rangle\langle m| .
$$

This mapping can be attained, alternatively, by solving the master equation corresponding to phase diffusion ${ }^{16}$. Quantum strategies aiming at an enhancement of the precision in phase estimation make use of N00N states, defined as $\frac{1}{\sqrt{2}}(|N 0\rangle+|0 N\rangle)$. Even under phase diffusion, the evolution of these states lies in the $2 \mathrm{D}$ space spanned by $|N, 0\rangle=\frac{1}{\sqrt{N !}}\left(\hat{a}^{\dagger}\right)^{N}$ $|00\rangle$ and $|0, N\rangle=\frac{1}{\sqrt{N !}}\left(\hat{b}^{\dagger}\right)^{N}|00\rangle$. A 2D picture also describes classical phase estimation strategies relying on coherent states. Indeed, a split coherent state with amplitude $\alpha$ yields the same precision as a collection of $|\alpha|^{2}$ independent split single photons (that is, N00N states with $N=1$ ). In these relevant cases, our twomode probe state can be effectively modelled as a single qubit

$$
\rho_{0}=\left(\begin{array}{cc}
\cos ^{2}\left(\frac{\theta}{2}\right) & \cos \left(\frac{\theta}{2}\right) \sin \left(\frac{\theta}{2}\right) \\
\cos \left(\frac{\theta}{2}\right) \sin \left(\frac{\theta}{2}\right) & \sin ^{2}\left(\frac{\theta}{2}\right)
\end{array}\right),
$$

which, acted upon by a phase shift $\phi$ and a phase diffusion channel parametrized by $\Delta$, yields

$$
\rho=\left(\begin{array}{cc}
\cos ^{2}\left(\frac{\theta}{2}\right) & \cos \left(\frac{\theta}{2}\right) \sin \left(\frac{\theta}{2}\right) e^{-i \phi-\Delta^{2}} \\
\cos \left(\frac{\theta}{2}\right) \sin \left(\frac{\theta}{2}\right) e^{i \phi-\Delta^{2}} & \sin ^{2}\left(\frac{\theta}{2}\right)
\end{array}\right) .
$$


The QFI matrix corresponding to parameters $\phi$ and $\Delta$, depending on the probe parameter $\theta$ can be calculated, using the SLD.

$$
\mathbf{H}_{\theta}(\phi, \Delta)=\mathbf{H}_{\theta}(\Delta)=\sin ^{2} \theta\left(\begin{array}{cc}
e^{-2 \Delta^{2}} & 0 \\
0 & \frac{4 \Delta^{2}}{e^{2 \Delta^{2}}-1}
\end{array}\right) .
$$

The maximum QFI corresponds to equatorial states with $\theta=\pi / 2$. From now on we shall refer to the diagonal elements of the matrix $\mathbf{H}_{\pi / 2}(\Delta)$ as $H_{11}$ and $H_{22}$. For N00N states and for coherent states with amplitude $\alpha$, the QFI matrices read

$$
\begin{aligned}
\mathbf{H}^{(\mathrm{NOON})}(\Delta) & =N^{2} \mathbf{H}_{\pi / 2}(N \Delta), \\
\mathbf{H}^{(\mathrm{coh})}(\Delta) & =|\alpha|^{2} \mathbf{H}_{\theta}(\Delta),
\end{aligned}
$$

respectively.

The SLDs corresponding to the two parameters do not commute. However, for equatorial states (corresponding to balanced interferometers), the expectation value of their commutator vanishes, that is, $\operatorname{Tr}\left[\rho\left(L_{1} L_{2}-L_{2} L_{1}\right)\right]=0$ for $\theta=\pi / 2$. In principle, when this condition is satisfied, a measurement that attains the QFI for joint estimation of both parameters can be constructed $^{31}$. This requires a collective measurement on multiple copies of evolved probe states, which is a challenging task to implement. Therefore, we firstly restrict our search for an optimal strategy to separable positive operator-valued measurements (POVMs) that is, measurements that act on probe states individually. We discuss extensions to joint measurements subsequently.

Trade-off in the estimation precision for $\phi$ and $\Delta$. In order to assess the performances of these measurements we consider the quantities $F_{1,1} / H_{1,1}$ and $F_{2,2} / H_{2,2}$, that is, the ratios between the FI and QFI values for $\phi$ and $\Delta$. Finding a relation between these ratios would effectively express the interplay that exists between the estimator variances corresponding to the two parameters. As shown in Supplementary Note 1, a trade-off relation can be derived, which is obeyed for all probe states and separable measurements:

$$
\frac{F_{1,1}}{H_{1,1}}+\frac{F_{2,2}}{H_{2,2}} \leq 1
$$

The most naive bound for the quantity in Equation (9) is equal to 2 , and it would be in principle achievable by means of a measurement that is optimal for both parameters. With this inequality, we not only prove that such measurement does not exist but we also quantify the maximum precision achievable in a joint estimation. Specifically, we prove that any measurement that is independently optimum for the estimation of one of the parameters is completely insensitive to the other. This bound is saturated by all POVMs with elements in the equatorial plane of the Bloch sphere, which have the form

$$
\Pi_{j}=\frac{n_{j}}{2}\left(\begin{array}{cc}
\frac{1}{2} & \frac{1}{2} e^{-\mathrm{i} \chi_{j}} \\
\frac{1}{2} e^{\mathrm{i} \chi_{j}} & \frac{1}{2}
\end{array}\right),
$$

with $0<n_{j}<1, \quad 0 \leq \chi_{j} \leq 2 \pi$, where the probability of outcome $j$ is $\operatorname{Tr}\left[\rho \Pi_{j}\right]$ and $\sum_{j} \Pi_{j}=\mathbb{I}$.

A further bound on statistical variances can be derived from this relation and Equation (1). The expected variance of the phase shift estimator obeys $\gamma_{1,1}=\operatorname{Var}(\phi) \geq\left[M\left(F_{1,1}-F_{1,2}^{2} / F_{2,2}\right)\right]^{-1}$ and an analogous relation can be written for the phase diffusion amplitude. Using the fact that the off-diagonal elements of the FI matrix are real numbers, we get $\operatorname{Var}(\phi) \geq\left(M F_{1,1}\right)^{-1}$ and $\gamma_{2,2}=$ $\operatorname{Var}(\Delta) \geq\left(M F_{2,2}\right)^{-1}$. Notice that the off-diagonal elements of the FI matrix correspond to the coupling of estimators for the two parameters, which results in increased statistical errors. Thus, the statistical variances obey

$$
\frac{H_{1,1}^{-1}}{\operatorname{Var}(\phi)}+\frac{H_{2,2}^{-1}}{\operatorname{Var}(\Delta)} \leq M
$$

This inequality is one of our main results. It is saturated when the inequality given in Equation (9) is saturated and the offdiagonal elements of the FI matrix are zero.

An optimal measurement. In Supplementary Note 1, we show that the bound in Equation 11 can be saturated for POVMs in the equatorial plane that are symmetric with respect to the measured state-meaning that for each operator of the form given in Equation (10), parametrized by $n_{j}=n$ and $\chi_{j}=\phi+\delta$, the POVM set contains another element, parametrized by $n_{j^{\prime}}=n$ and $\chi_{j^{\prime}}=$ $\phi-\delta$ for some $\delta$. Note that in general the POVM saturating the bound depends on the specific value of the phase $\phi$.

We prove in Supplementary Note 2 that a double homodyne set-up, combining modes $a$ and $b$ on a beam splitter and measuring the $X$ and $P$ quadratures, respectively, in the beam splitter's two outputs, saturates the bound in Equation (11) independently of the value of $\phi$. Figure 1 shows the dependence of the variances on $\Delta$ for this set-up, which is depicted in Supplementary Fig. 1.

Experiment. We adopt our theory to quantify the effectiveness of an actual experimental set-up for joint parameter estimation for
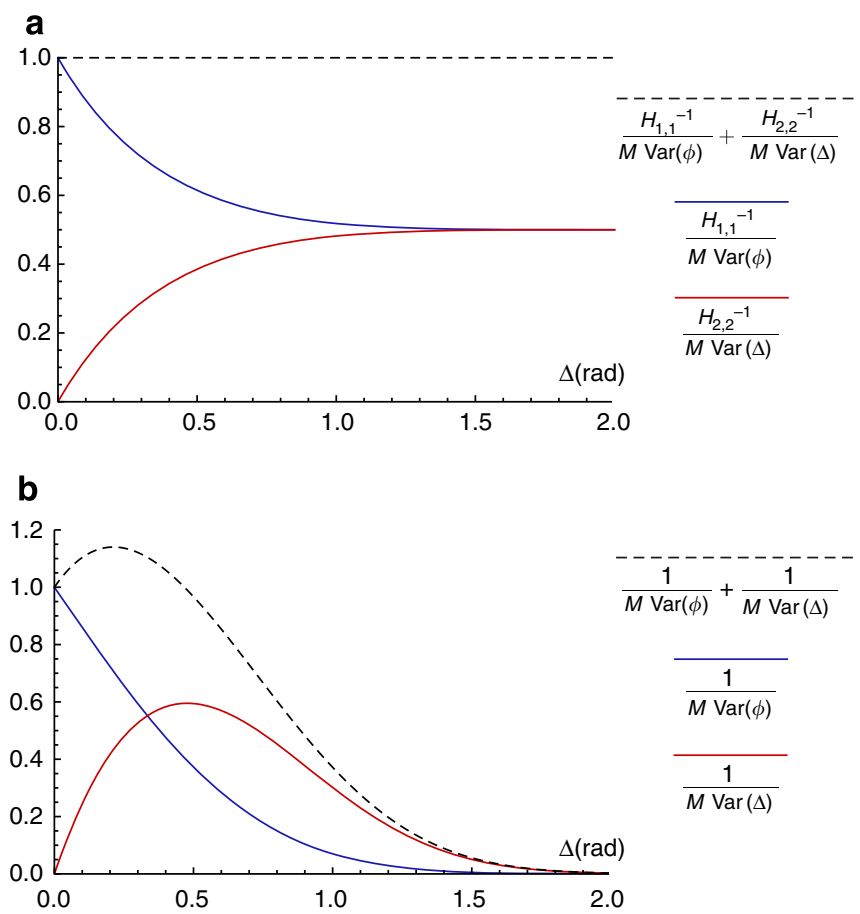

Figure 1 | Joint parameter estimation by double homodyne. (a) The ratios between optimum single parameter statistical variances and statistical variances that can be achieved with the double homodyne set-up, as a function of phase diffusion amplitude, for a split single photon probe state (first order NOON state). Note that, for low phase diffusion amplitude, the homodyne set-up measures phase optimally. (b) Fl elements, for a split single photon. Blue corresponds to phase estimation precision and red to phase diffusion amplitude estimation precision, while black corresponds to the sum of the two. For NOON states with $\mathrm{N}$ photons, plots a and b scale by a factor of $N^{2}$ vertically and by a factor of $1 / N$ horizontally. A schematic of this measurement set-up, which implements a continuous measurement, is included as Supplementary Fig. 1. 
polarimetry by investigating how close the implementation compares with the optimal bound in Equation (11). We are not aiming at demonstrating a quantum advantage and realize our implementation with coherent states. The joint estimation of phase and phase diffusion requires a measurement with at least three outputs. This is because the FI matrix corresponding to any single qubit (two-output) projective measurement is singular. Thus, it cannot be inverted, yielding unbounded estimator variances. We implemented a four-outcome measurement based on a displaced Sagnac polarization interferometer ${ }^{33,37}$ depicted in Fig. 2. Our measurement realizes a mixture of the optimal projective measurements for estimating the phase and the phase diffusion amplitude, respectively. The set-up can be arranged to tune the different weights of these measurements by rotating a waveplate.

Complete information about the POVM associated to each of these measurements is obtained via detector tomography ${ }^{38}$. This technique adopts a quorum of input states and records the probabilities of the outcomes. The Born rule then allows to reconstruct the measurement operator. These reconstructed POVMs are then used to compute the relevant FI matrix, as detailed in the Methods section. In Fig. 3 we report our results, where the variances $\operatorname{Var}(\phi)$ and $\operatorname{Var}(\Delta)$ have been estimated from the classical Cramér-Rao bound. The plot is obtained by varying the measurement from $\sigma_{x}$, the optimal measurement for phase estimation, to $\sigma_{y}$, the optimal measurement for estimating the phase diffusion amplitude.

The experimental results are close to the optimum precision given by Equation (11), with the imperfections of the implementation stemming mainly from no-unit interference visibility and imperfect alignment of the set-up. The precision for the estimates of $\phi$ depends strongly on the measurement visibility

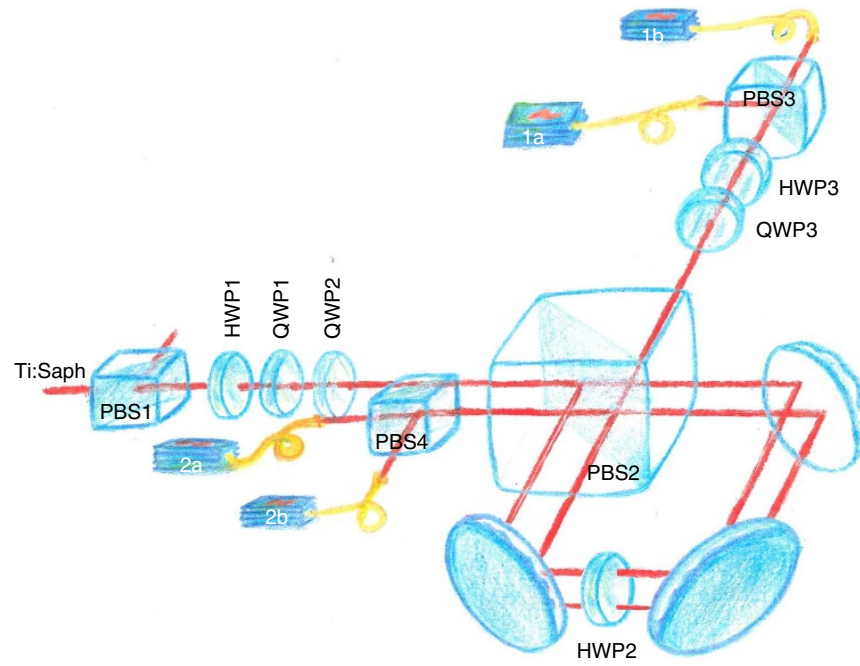

Figure 2 | Experimental set-up. In our set-up, the two modes $a$ and $b$ correspond to the horizontal $(H)$ and vertical $(V)$ polarizations of a single spatial mode. In this basis, the diagonal polarization states are defined as $|D\rangle=\frac{1}{\sqrt{2}}(|H\rangle+|V\rangle)$ and $|A\rangle=\frac{1}{\sqrt{2}}(|H\rangle-|V\rangle)$ and the circular polarization states as $|R\rangle=|H\rangle+i|V\rangle$ and $|L\rangle=|H\rangle-i|V\rangle$. The four outputs of the polarization interferometer correspond approximately to the following POVM operators acting on the input polarization state: $\prod_{1 a}=k|D\rangle\left\langle D\left|, \prod_{1 b}=k\right| A\right\rangle\left\langle A\left|, \prod_{2 a}=(1-k)\right| R\right\rangle\langle R|, \prod_{2 a}=(1-k)$ $|L\rangle\langle L|$, where $k$ is a tunable parameter. This measurement should saturate the inequality given in Equation 11 for certain input states. PBS, polarizing beam splitter; HWP, half-wave plate; QWP, quarter-wave plate. corresponding to outputs $1 a$ and $1 b$ (according to Fig. 3). For $\Delta$, the precision strongly depends on the visibility corresponding to outputs $2 a$ and $2 b$. The influence of non-unit visibility is more pronounced for the latter, as we detail in the Supplementary Methods.

Extensions. We have so far restricted both theoretical and experimental studies to measurements on single quantum probes. Collective measurements on multiple copies of probe states may get closer to the multiparameter quantum Cramér-Rao bound in some cases $^{31}$. We study this for the simplest nontrivial case, that of an entangled projective measurement on a pair of qubit probe states that have undergone the same phase shift and phase diffusion. In Supplementary Note 3, we analyse the performance of a Bell measurement (in the basis in which the states of Equation (5) are written).

In such a set-up, the Bell measurement can perform joint estimation with precision surpassing the bound established in

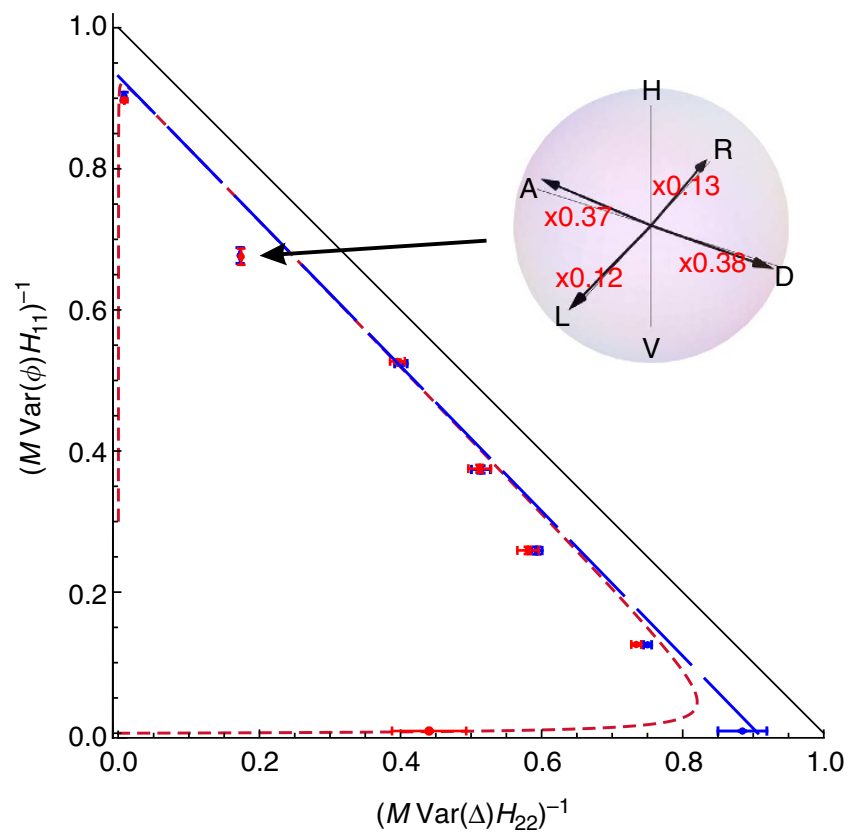

Figure 3 | Experimental results. Parametric plot of the estimates for the ratios between optimum single parameter statistical variances and optimum statistical variances achievable with our experimental set-up (details in the Methods section). Blue points are calculated for a phase shift of approximately $\frac{\pi}{2^{\prime}}$ optimized to obtain null off-diagonal elements of the $\mathrm{FI}$ matrix. Red points are calculated for a phase shift differing by $1^{\circ}$ with respect to the one corresponding to the blue points. The black line gives the ultimate limit. The blue and red dashed lines give the theoretical prediction for the blue and red points, respectively, assuming visibilities of $96.5 \%$ for outputs $1 \mathrm{a}$ and $1 \mathrm{~b}$ and $99.4 \%$ for outputs $2 \mathrm{a}$ and $2 \mathrm{~b}$. Insert: Bloch sphere representation of the estimated POVM operators, for the indicated setting (all the estimated POVMs are represented in Supplementary Fig. 2). The vectors represent the measurement operators corresponding to the four outputs, each normalized. The numbers written on the vectors are the trace norms of the corresponding operators, weighed by the total trace of the four operators. We choose a phase diffusion amplitude $\Delta=0.25 \mathrm{rad}$ $\left(\approx 14^{\circ}\right)$. Error bars are obtained from a Monte-Carlo simulation with 500 repetitions, with random variations of the measured output intensities, distributed according to measured fluctuations. Their length corresponds to two standard deviations. Details on how this figure is obtained are presented in the Supplementary Methods. 


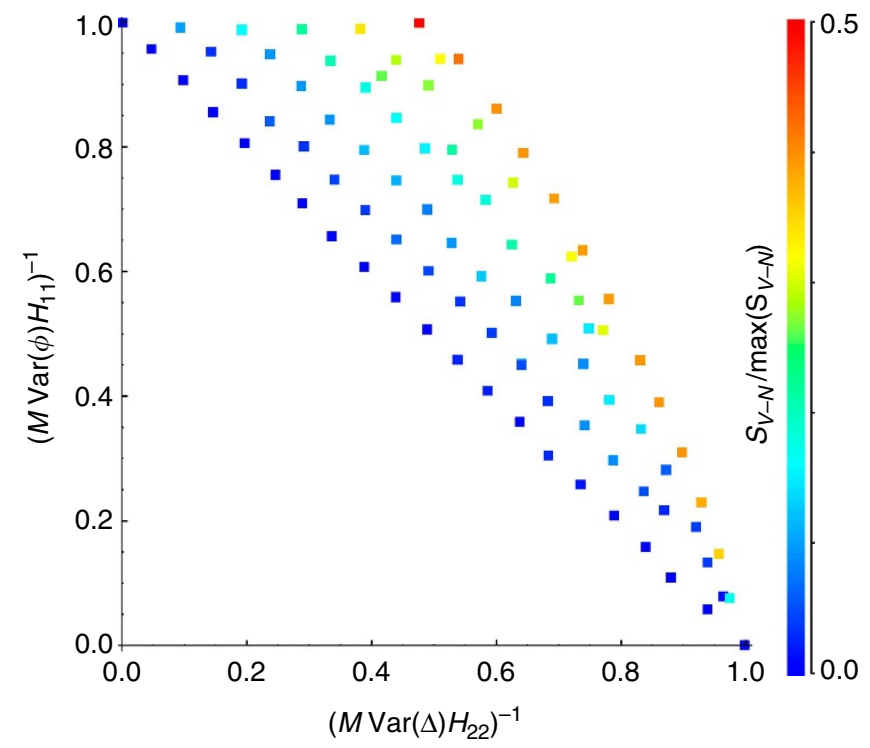

Figure 4 | Collective measurements. Results of a simulated annealing search over all projectors acting on the space of two qubit probe states. Points are shown for all coordinates that signify a violation of the bound given by Equation (11). Color illustrates the smallest value of total entropy of entanglement of the projectors found by the search in the corresponding area, weighed by the maximum possible value (which corresponds to a Bell measurement). The maximum sum of coordinates in this graph is 1.48 and the corresponding entropy of entanglement is 0.425 . The search is performed for a phase diffusion amplitude $\Delta=0.25 \mathrm{rad}\left(\approx 14^{\circ}\right)$. The details of the search are presented in the Methods section and Supplementary Methods.

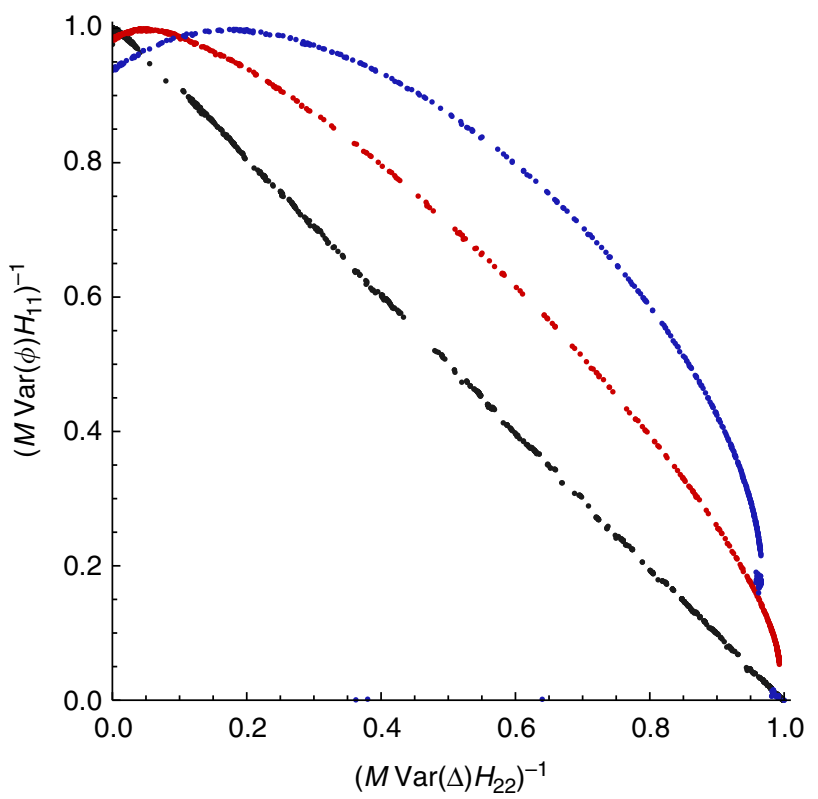

Figure 5 | Joint estimation using HB states. Limits found by a simulated annealing search over all projectors acting on the space of the $\mathrm{HB}(3)$ state. The black points correspond to $\Delta=0.01 \mathrm{rad}\left(\approx 0.6^{\circ}\right)$, red points to $\Delta=0.05 \mathrm{rad}$ and blue points to $\Delta=0.1 \mathrm{rad}$. The details of the search are presented in the Methods section, in Supplementary Fig. 3 and in the Supplementary Methods.

Equation (11) for separable measurements, as long as the amplitude of phase diffusion is less than $\Delta_{0}$, corresponding to $e^{-\Delta_{0}^{2}}=\sqrt{2 /(1+\sqrt{5})}$. Indeed, for $\Delta=0$, a Bell measurement yields

$$
\frac{H_{1,1}^{-1}}{\operatorname{Var}(\phi)}+\frac{H_{2,2}^{-1}}{\operatorname{Var}(\Delta)}=\frac{3}{2} M,
$$

a value larger than the right side of the inequality given by Equation (11), implying that greater precision can be obtained by investing in collective measurements.

For a larger value of phase diffusion, we performed a numerical search over all two qubit projective measurements, yielding the achievable pairs of $\left\{\frac{H_{1,1}^{-1}}{M \operatorname{Var}(\phi)}, \frac{H_{2,2}^{-1}}{M \operatorname{Var}(\Delta)}\right\}$ and also optimizing for the smallest total of the entropy of entanglement for the corresponding projectors. Figure 4 shows the results of this numerical search, revealing how a higher violation of the bound derived for separable measurements can be obtained with a more entangled measurement.

Our trade-off relations have been derived for those states whose evolution is effectively described in a $2 \mathrm{D}$ Hilbert space. In order to explore the form that this trade-off takes with probe states in larger spaces, we present a numerical study of the performance of Holland-Burnett (HB) states ${ }^{2}$. These provide the same precision scaling as $\mathrm{N} 00 \mathrm{~N}$ states, but are more resilient to losses than the latter. $A n \operatorname{HB}(N)$ state results from the interference of two $\mathrm{N}$-photon modes on a beam splitter.

We performed a numerical search over all projective measurements on the $4 \mathrm{D}$ space corresponding to the $\mathrm{HB}(3)$ state,

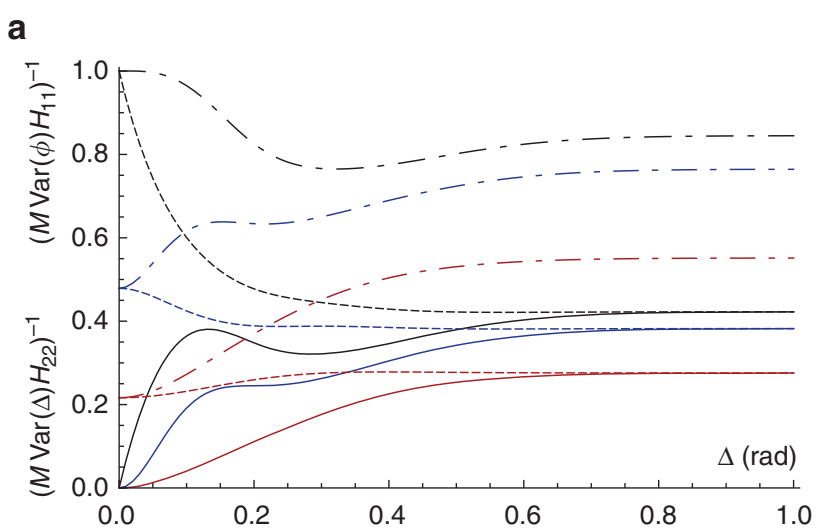

b

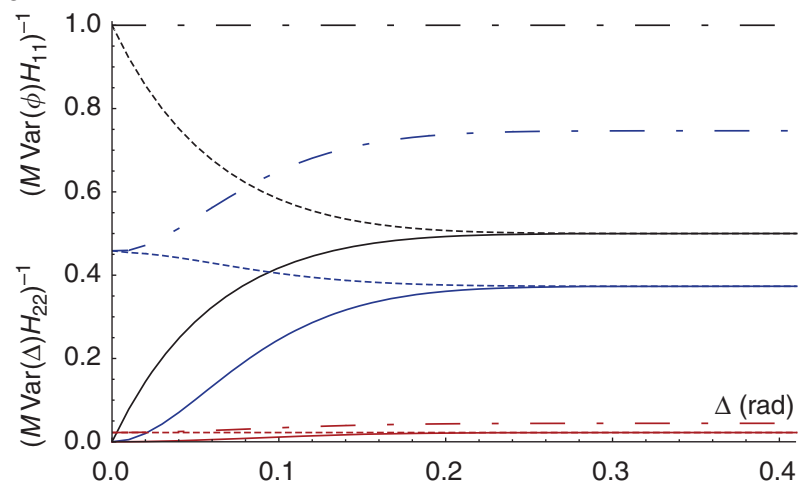

Figure 6 | Practical set-up with losses. The ratios between the optimum single parameter statistical variances and statistical variances that can be achieved with a double homodyne measurement; solid line for phase diffusion estimation, dashed line for phase estimation and dot-dashed line for the sum of the two; (a) for an $\mathrm{HB}(3)$ probe state (with 6 photons) and (b) for a NOON(6) probe state; with symmetric losses-black for unit efficiency, blue for 0.95 total efficiency and red for 0.5 total efficiency. Note that the $\mathrm{HB}$ state is better suited for parameter estimation with loss than the NOON state. 
optimizing the set of values $\left\{\frac{H_{1,1}^{-1}}{M \operatorname{Var}(\phi)}, \frac{H_{2,2}^{-1}}{M \operatorname{Var}(\Delta)}\right\}$. The trade-off bounds observed in the results of the search depend on the amplitude of the phase diffusion. While for $\Delta=0$, the linear trade-off expressed by Equation (11) is observed, for larger phase diffusion, we obtain limits higher than this (results are presented in Fig. 5). In Supplementary Fig. 4, we show how a photon number resolving measurement ${ }^{12}$ can beat the limit in Equation (11) when applied to HB states, however not reaching the bounds depicted in Fig. 5 .

It is recognized that the precision of any measurement making use of entangled states is affected by loss. Here we illustrate a different effect of loss, that is, how it affects the performance of simultaneous estimation. We focus on a practical scenario where the double homodyne measurement is used to analyse $\mathrm{HB}$ and N00N states with six photons; the results are shown in Fig. 6. They illustrate the fact that $\mathrm{HB}$ states are more robust to loss than N00Nstates not only in terms of QFI scaling, but also for attaining a satisfactory joint estimation precision.

We demonstrate in Supplementary Note 4 that for all pathsymmetric probe states (of which $\mathrm{HB}$ states are an example), with $\Delta=0$ and no loss, the double homodyne measurement estimates phase optimally 39,40 . In our results (presented in Fig. 6), the decrease in sensitivity due to loss is partly contained in the decreasing value of the QFI. In addition, the classical FI corresponding to double homodyne detection degrades with respect to the QFI due to the effect of the incoherent part of the loss-affected probe signal, which introduces noise in the measurement outcomes (this is detailed in Supplementary Fig. 5).

\section{Discussion}

Figure 3 shows the variances that can be obtained in our experimental set-up with a probe state that has a phase shift of $1^{\circ}$ with respect to the optimal probe state. We highlight a somewhat overlooked aspect of parameter estimation: the sensitivity of the measurements to experimental imperfections in the alignment of the phase of the probe state. For a large extent of the settings of our tunable measurement, the precision of the estimates is robust to this small variation in phase. This is because the four-outcome POVM is capable of distinguishing between the rotation and the shrinking of the Bloch vector corresponding to the probe state. As we tune the measurement close to either of the extremal points, corresponding to $\left\{\sigma_{x}, \sigma_{y}\right\}$, this ability is compromised. While with a projective measurement ( $\sigma_{x}$ or $\sigma_{y}$ for our set-up), when there is no prior information on the amplitude of the phase diffusion, phase estimation is not possible, with a balanced setting of the weights given to pairs of projectors, our set-up is tolerant to phase alignment (this is illustrated in Supplementary Fig. 6 and the Supplementary Methods). Notably, the performance of the double homodyne set-up described in this work is completely independent of the phase of the probe state.

We have applied our study to quantum correlated states that offer enhanced sensitivity for phase estimation. We have also shown that collective measurements can offer an advantage for joint estimation. However, entangled measurements in optics require either probabilistic schemes, which have limited applicability in metrology, or strong nonlinearities, which may be challenging and at the edge of current technology.

We have found that states with correlations over multiple Fock layers, such as HB states, can perform better than N00N states in terms of joint estimation.

\section{Methods}

Experimental set-up. The source is a mode-locked Ti:sapphire laser, working in the pulsed regime, with central wavelength of $830 \mathrm{~nm}$, bandwidth of $32 \mathrm{~nm}$ and a repetition rate of $256 \mathrm{kHz}$. The preparation stage consists of a polarizing beam splitter (PBS1) that transmits only horizontally polarized light, followed by a half-wave plate (HWP1) and a quarter-waveplate (QWP1), used for the preparation of polarization states for detector tomography. QWP2 is set at $45^{\circ}$, rotating $|R\rangle$ to $|V\rangle$ and $|L\rangle$ to $|H\rangle$. The displaced Sagnac interferometer consists of two slightly displaced counter-propagating modes of equal length. The input state is split by PBS2 into its $|H\rangle$ and $|V\rangle$ components, corresponding to the two paths of the interferometer. After being acted upon by HWP2, the two paths recombine on PBS2. Depending on the orientation of HW2, the input beam is split and directed towards outputs 1 and 2 . The polarization state at output 1 is approximately that of the input with a phase shift due to a path difference in the arms of the interferometer. QWP3 is a multiorder waveplate, with axis vertical, which is twisted in order to correct for this phase shift. The displaced Sagnac interferometer acts as a tunable non-polarizing beam splitter, with the added effect of switching $|H\rangle$ and $|V\rangle$ polarizations in output 2. The detectors situated after HWP3 and PBS3 measure polarizations $|D\rangle$ and $|A\rangle$, respectively. The detectors situated after PBS4 measure $\{|H\rangle,|V\rangle\}$ in output 2, effectively measure part of the input polarization state in the basis $\{|R\rangle,|L\rangle\}$. Single-mode fibres are used to couple light into the detectors and alignment of the interferometer is performed by coupling the horizontal and vertical modes independently into single-mode fibres. The interferometer phase is set so that a minimum of interference is measured in output $1 a$ when the input polarization state is $|D\rangle$. The measured visibility of the interference was $\sim 97 \%$.

We characterized the tunable measurement by performing detector tomography, with different settings of HWP3, and measuring intensities with a photodiode.

Estimation and errors. The experimental errors affecting our set-up are the following: statistical errors intrinsic to quantum measurement, which are the object of our study; loss and distinguishability of photons, which are accounted for in the description of the set-up, and technical (systematic) errors. The latter dominate statistical errors in our characterization of the set-up. One of the two easy-toidentify error sources consists of intensity fluctuations on a timescale longer than the detection time, which can be dealt with by recording traces of the intensity readings and using the measured distributions when fitting data to the POVM model. The second consists of imperfections in the manufacturing and calibration of the waveplates used for preparation of the input polarization state.

The POVMs are estimated by using a maximum likelihood algorithm comparing the collected data with the predictions from the reconstruction. We verify that the outcomes predicted by the reconstructed POVMs differ from those measured by values accountable for by observed fluctuations. The error bars for the estimated FI are computed using a Monte Carlo simulation, starting with the variance of the measured values of light intensities. More detailed information on how Fig. 3 was obtained is present in the Supplementary Methods.

Searches over projective measurements. All elements of the set of projective measurements in a $d$-dimensional Hilbert space can be produced by acting on an orthonormal basis of this space with a unitary transformation. We perform simulated annealing ${ }^{41}$ over the set of projective measurements by using random unitary transformations to perform a random walk. The algorithm decides whether a step is made in a randomly generated direction according to a tunable distribution that favours increasing values of $\frac{H_{1,1}^{-1}}{M \operatorname{Var}(\phi)}$ and $\frac{H_{2,2}^{-1}}{M \operatorname{Var}(\Delta)}$ and, for the search presented in Fig. 4, decreasing values of the total entropy of entanglement of the projectors. We modify the step size, as well as the distribution controlling the random walk in order to reach the extreme values of the parameters that we are interested in, while ensuring that local minima are avoided. Details of this method, as well as arguments to restrict our search to projective measurements are presented in the Supplementary Methods.

\section{References}

1. Giovannetti, V., Lloyd, S. \& Maccone, L. 'Quantumenhanced measurements: beating the standard quantum limit'. Science 306, 1330-1336 (2004).

2. Holland, M. J. \& Burnett, K. Interferometric detection of optical phase shifts at the Heisenberg limit. Phys. Rev. Lett. 71, 1355-1358 (1993).

3. Bollinger, J. J., Itano, W. M., Winel, D. J. \& Heinzen, D. J. Optimal frequency measurements with maximally corre-lated states. Phys. Rev. A 54, 4649-4652 (1996).

4. Giovannetti, V., Lloyd, S. \& Maccone, L. Quantum metrology. Phys. Rev. Lett 96, 010401 (2006).

5. Nagata, T., Okamoto, R., O’Brien, J. L., Sasaki, K. \& Takeuchi, S. Beating the standard quantum limit with four-entangled photons. Science 316, 726-729 (2007).

6. Afek, I., Ambar, O. \& Silberberg, Y. High-noon states by mixing quantum and classical light. Science 328, 879-881 (2010).

7. Yonezawa, H. et al. Quantum-enhanced optical-phase tracking. Science 337, 1514-1517 (2012).

8. Simmons, S., Jones, J. A., Karlen, S. D., Ardavan, A. \& Morton, J. J. L. Magnetic field sensors using 13-spin cat states. Phys. Rev. A 82, 022330 (2010). 
9. Luff, B. J. et al. Integrated optical mach-zehnder biosensor. J. Lightwave Technol. 16, 583 (1998).

10. Crespi, A. et al. Measuring protein concentration with entangled photons. Appl. Phys. Lett. 100, 233704 (2012).

11. Demkowicz-Dobrzanski, R., Kolodynski, J. \& Guta, M. The elusive Heisenberg limit in quantum-enhanced metrology. Nat. Commun. 3, 1063 (2012).

12. Datta, A. et al. Quantum metrology with imperfect states and detectors. Phys. Rev. A 83, 063836 (2011).

13. Demkowicz-Dobrzanski, R. et al. Quantum phase estimation with lossy interferometers. Phys. Rev. A 80, 013825 (2009).

14. Dorner, U. et al. Optimal quantum phase estimation. Phys. Rev. Lett. 102 , 040403 (2009).

15. Kacprowicz, M., Demkowicz-Dobrzanski, R., Wasilewski, W., Banaszek, K. \& Walmsley, I. A. Experimental quantum-enhanced estimation of a lossy phase shift. Nat. Photon. 4, 357-360 (2009).

16. Brivio, D. et al. Experimental estimation of one-parameter qubit gates in the presence of phase diffusion. Phys. Rev. A 81, 012305 (2010).

17. Genoni, M. G., Olivares, S. \& Paris, M. G. A. Optical phase estimation in the presence of phase diffusion. Phys. Rev. Lett. 106, 153603 (2011).

18. Genoni, M. G. et al. Optical interferometry in the presence of large phase diffusion. Phys. Rev. A 85, 043817 (2012).

19. Escher, B. M., Davidovich, L., Zagury, N. \& de Matos Filho, R. L. Quantum metrological limits via a varia- tional approach. Phys. Rev. Lett. 109, 190404 (2012).

20. Knysh, S. I. \& Durkin, G. A. Estimation of Phase and Diffusion: Combining Quantum Statistics and Classical Noise. Preprint at http://arxiv.org/abs/ 1307.0470 (2013).

21. Yuen, H. P. \& Lax, M. Multiple-parameter quantum estimation and measurement of nonselfadjoint observables. IEEE Trans. Inform. Theory 19, 740-750 (1973)

22. Helstrom, C. W. \& Kennedy, R. S. Noncommuting observables in quantum detection and estimation theory. IEEE Trans. Inform. Theory 20, 16-24 (1974).

23. Belavkin, V. P. Generalized uncertainty relations and efficient measurements in quantum systems. Theoret. Math. Phys. 26, 213-222 (1976).

24. Chiribella, G., D’Ariano, G. M. \& Sacchi, M. F. Joint estimation of real squeezing and displacement. J. Phys. A Math. General 39, 2127 (2006).

25. Young, K. C., Sarovar, M., Kosut, R. \& Whaley, K. B. Optimal quantum multiparameter estimation and application to dipole- and exchange-coupled qubits. Phys. Rev. A 79, 062301 (2009).

26. Watanabe, Y., Sagawa, T. \& Ueda, M. Optimal measurement on noisy quantum systems. Phys. Rev. Lett. 104, 020401 (2010).

27. Monras, A. \& Illuminati, F. Information geometry of gaussian channels. Phys. Rev. A 81, 062326 (2010).

28. Monras, A. \& Illuminati, F. Measurement of damping and temperature: precision bounds in gaussian dissipative channels. Phys. Rev. A 83, 012315 (2011).

29. Crowley, P. J. D., Datta, A., Barbieri, M. \& Walmsley, I. A. A tradeoff in simultaneous quantum-limited phase and loss estimation in interferometry. Phys. Rev. A 89, 023845 (2014).

30. Vaneph, C., Tufarelli, T. \& Genoni, M.G. Quantum estimation of a two-phase spin rotation. Quantum Measure. Quantum Metrol. 1, 12-20 (2013).

31. Gill, R. D. \& Guta, M. On Asymptotic Quantum Statistical Inference. Phys. Rev. A. 89, 023845 (2014).

32. Genoni, M. G. et al. Optimal estimation of joint parameters in phase space. Phys. Rev. A 87, 012107 (2013).
33. Nagata, T., Okamoto, R., O’Brien, J. L., Sasaki, K. \& Takeuchi, S. Beating the standard quantum limit with four-entangled photons. Science 316, 726-729 (2007).

34. Cramer, H. Mathematical Methods of Statistics (Princeton University Press, 1946).

35. Helstrom, C. W. Quantum Detection and Estimation Theory (Academic Press, 1976).

36. Paris, M. G. A. Quantum estimation for quantum technology. Int. J. Quantum Inform. 7, 125 (2009).

37. Mosley, P. J., Croke, S., Walmsley, I. A. \& Barnett, S. M. Experimental realization of maximum confidence quan-tum state discrimination for the extraction of quantum information. Phys. Rev. Lett. 97, 193601 (2006).

38. Lundeen, J. S. et al. Tomography of quantum detectors. Nat. Phys. 5, 27-30 (2008).

39. Seshadreesan, K. P., Kim, S., Dowling, J. P. \& Lee, H. Phase estimation at the quantum cramer-rao bound via parity detection. Phys. Rev. A 87, 043833 (2013).

40. Plick, W. N., Anisimov, P. M., Dowling, J. P., Lee, H. \& Agarwal, G. S. Parity detection in quantum optical metrology without number-resolving detectors. New J. Phys. 12, 113025 (2010).

41. Kirkpatrick, S., Gelatt, C. D. \& Vecchi, M. P. Optimization by simulated annealing. Science 220, 671-680 (1983).

\section{Acknowledgements}

We thank Joshua Nunn, Tim Bartley, Mark Mitchison, David Jennings and Paolo Mataloni for discussion and comments. M.D.V. is supported by the EPSRC via the Controlled Quantum Dynamics CDT. X.M.J. is supported by an EU Marie-Curie Fellowship (PIIF-GA-2011-300820). WSK is supported by an EU Marie-Curie Fellowship (PIIF-GA-2011-331859). M.G.G. acknowledges a fellowship support from UK EPSRC (grant EP/I026436/1). M.S.K. is supported by the Qatar National Research Fund (NPRP4-5554-1-084). This work was supported by the EPSRC (EP/H03031X/1, EP/ K034480/1, EP/K026267/1), the European Commission project SIQS, the Air Force Office of Scientific Research (European Office of Aerospace Research and Development).

\section{Author contributions}

M.D.V. developed the theory, designed and performed the experiment. M.G.G. contributed to the theory. G.D., X.M.J. and W.S.K. contributed to the set-up and assisted with preliminary data collection. A.D. and M.B. conceived and supervised the project and helped with designing the experimental set-up. M.S.K. and I.A.W. supervised the project and contributed with discussion.

\section{Additional information}

Supplementary Information accompanies this paper at http://www.nature.com/ naturecommunications

Competing financial interests: The authors declare no competing financial interests.

Reprints and permission information is available online at http://npg.nature.com/ reprintsandpermissions/

How to cite this article: Vidrighin, M. D. et al. Joint estimation of phase and phase diffusion for quantum metrology. Nat. Commun. 5:3532 doi: 10.1038/ncomms4532 (2014). 